\title{
Storing images in warm atomic vapor
}

\author{
M. Shuker, ${ }^{1}$ O. Firstenberg, ${ }^{1}$ R. Pugatch, ${ }^{2}$ A. Ron, ${ }^{1}$ and N. Davidson ${ }^{2}$ \\ ${ }^{1}$ Department of Physics, Technion-Israel Institute of Technology, Haifa 32000, Israel \\ ${ }^{2}$ Department of Physics of Complex Systems, Weizmann Institute of Science, Rehovot 76100, Israel
}

\begin{abstract}
Reversible and coherent storage of light in atomic medium is a promising method with possible application in many fields. In this work, arbitrary two-dimensional images are slowed and stored in warm atomic vapor for up to $30 \mu \mathrm{s}$, utilizing electromagnetically induced transparency. Both the intensity and the phase patterns of the optical field are maintained. The main limitation on the storage resolution and duration is found to be the diffusion of atoms. A technique analogous to phase-shift lithography is employed to diminish the effect of diffusion on the visibility of the reconstructed image.
\end{abstract}

PACS numbers: 42.50.Gy, 32.70.Jz

When a resonant light pulse ("probe") impinges upon a gas of atoms, it is strongly absorbed, exciting the atoms to an upper state. However, if a second "pump" beam is present, which couples a second state to the same excited state, than the "probe" pulse will be able to pass the sample - a phenomenon known as electromagnetically induced transparency (EIT) [1]. The unique properties of EIT allow for a wide range of coherent light-matter phenomena, including non-linear optics 2], entanglement [3], generation of quantum pulses of light [4], and quantum communication [5]. In Ref. [6], the group velocity of the probe pulse, in a medium of ultra-cold atoms, was decreased to $17 \mathrm{~m} / \mathrm{s}$ and similar results were achieved in warm vapor [7]. In Refs. [8, 9, 10], it was demonstrated that the probe pulse can be completely stopped, while it is contained inside the medium, by shutting off the pump. The pulse can be recovered by reopening the pump beam after a certain "storage duration". A prominent feature of this technique is the reversible and coherent storage of the information carried by the probe, in the atomic coherences.

Here, we report a method for reversibly capturing complex three-dimensional light fields using EIT in atomic vapor [1, 8]. The storage experiments discussed above have utilized a transverse Gaussian mode for both pump and probe beams. The current research is focused on slowing and storing information imprinted in the transverse plane of the probe beam ("images"), and on reducing the effects of atomic diffusion. In a previous work [11], the ability to slow images and delay them for several ns was demonstrated, using dispersion from fardetuned absorption lines. In the current work, we use EIT to slow images to a group velocity of several thousands $\mathrm{m} / \mathrm{s}$, achieving delays of several $\mu \mathrm{s}$. We further use the unique properties of EIT to store the images in the atomic medium for a similar duration. The long slowing delays and storage durations are comparable with the typical diffusion time in which atoms cross the image. We demonstrate the deteriorating effect of diffusion by storing images of digits for different durations. Finally, we introduce a technique to diminish the effect of atomic diffusion by alternating the phase of neighboring features. This technique, which is the atomic analogue of the optical phase-shift lithography [12], is demonstrated by storing an image of three lines and studying the effect of flipping the phase of adjacent lines.

Slowing of images may prove useful for various image processing and image-correlation applications. An intriguing possibility is to use the diffusion during slowing or storage as a complex diffusion filter, thus realizing an all optical edge detector [13]. In the context of quantum information processing, a single-cell device for multiqubit memory can be implemented, e.g., by a spatial array of optical qubits in the transverse plane. Storing images, rather than slowing them, has the fundamental advantages of supporting longer delays and completely converting the optical information to atomic coherence, hence making it more amenable to various methods of manipulation, e.g., phase conjugation [14].

The storage experiments were performed within the $\mathrm{D} 1$ transition of ${ }^{87} \mathrm{Rb}[10$ as depicted in Fig. 1.a. Two Zeeman sub-levels of the ground state $\left(\left|F=2 ; m_{F}=0\right\rangle,\left|F=2 ; m_{F}=+2\right\rangle\right)$ are used as the two lower levels of a $\Lambda$-system. The experimental setup is depicted in Fig. 1.b. An external cavity diode laser (ECDL) is stabilized to the $F=2 \rightarrow F^{\prime}=1$ transition. The laser is divided into two beams of perpendicular polarizations, the pump and the probe, using a polarizing beam-splitter (PBS). The pump and the probe pass through acousto-optic modulators (AOM), allowing us to precisely control the Raman detuning. The pump beam is shaped as a large Gaussian beam with a waist radius of $w_{\text {pump }}=2.1 \mathrm{~mm}$ and a total intensity of 5.8 $\mathrm{mW}$. The probe beam is shaped as a Gaussian beam with a waist radius of $w_{\text {probe }}=1 \mathrm{~mm}$ and intensity of 200 $\mu \mathrm{W}$ and passes through a binary image mask, which is a standard resolution target. The plane of the imagemask is imaged onto the center of the vapor cell using two lenses (the optical design and the feature size of the images insure that the diffraction along the cell's length is negligible). The pump and the probe are recombined on a second PBS and co-propagate towards the cell. A quar- 
ter wave-plate before the cell converts the pump and the probe polarizations to $\sigma^{+}$and $\sigma^{-}$respectively. We use a $5 \mathrm{~cm}$ long vapor cell, containing isotopically pure ${ }^{87} \mathrm{Rb}$ and 10 Torr of Neon buffer gas. Frequent collisions with the buffer gas induce Dicke-like narrowing 15, increasing the acceptance angle of the probe beam [16, 17], thus allowing for smaller features in the transverse plane. The diffusion coefficient of $\mathrm{Rb}$ atoms is determined from independent measurements to be $D=10 \mathrm{~cm}^{2} / \mathrm{s}$. The temperature of the cell is $\sim 52^{\circ} \mathrm{C}$, providing a Rubidium vapor density of $\sim 1.3 \times 10^{11} / \mathrm{cc}$. The cell is placed within a four-layered magnetic shield, and a set of Helmholtz coils provides a small, $B_{z}=50 \mathrm{mG}$, axial magnetic field to set the quantization axis.

The experimental sequence of the storage experiments is detailed in Fig. 1.c. A pulse of the probe beam (Gaussian; $\sigma=5 \mu \mathrm{s}$ ) travelled in the EIT medium with a group velocity of $\sim 8000 \mathrm{~m} / \mathrm{s}$ - resulting in a slowly propagating image [11]. The images were stored in the medium by turning off the pump after about half of the probe pulse had exit the cell. After an arbitrary storage duration, the pump beam was turned on, and the stored image reappeared. Upon exiting the cell, the beams were separated using polarization optics, and the probe beam was imaged onto a CCD camera, whose focus was adjusted to the exit plane of the cell. The camera trigger was set to measure only the restored part of the probe beam (see Fig. 1.c), and special care is taken to avoid the detection of the leaked part of the probe. As a proofof-principle, we stored images of the digits '2', '6', and ' 9 ' in the atomic vapor. The images were created using a standard resolution target as a mask, and by shifting the mask to imprint different digits in different measurements. Fig. 2 shows the images without slowing (left column), with slowing only (center column) and after being stored and retrieved (right column). Evidently, the image of the digit '2', which was slowed for $6 \mu \mathrm{s}$, was smoothed, and its sharp edges were softened (similar images were achieved for the other digits). We attribute this effect to atomic diffusion during the slow propagation of the dark-state polariton 18]. The diffusion effect is pronounced due to the sharp and narrow features in the image and due to the significantly larger delay time, compared with a previous demonstration of slow images in hot vapor [11]. The lower signal-to-noise ratio in the slowed image is mainly due to the lower transmission, compared with the off-resonance image.

The images of the digits '2',' 6 ', and '9' were stored for 2,6 , and $9 \mu \mathrm{s}$, respectively. During the storage, the images are encoded in the quantum state of the atomic ensemble [8] and thus susceptible to degradation through various relaxation processes, such as spin-exchange, collisions, and diffusion. Nevertheless, the main features survive through the storage process, and the digits are still identifiable. Two degradation effects are evident from the stored images: broadening of the image features due

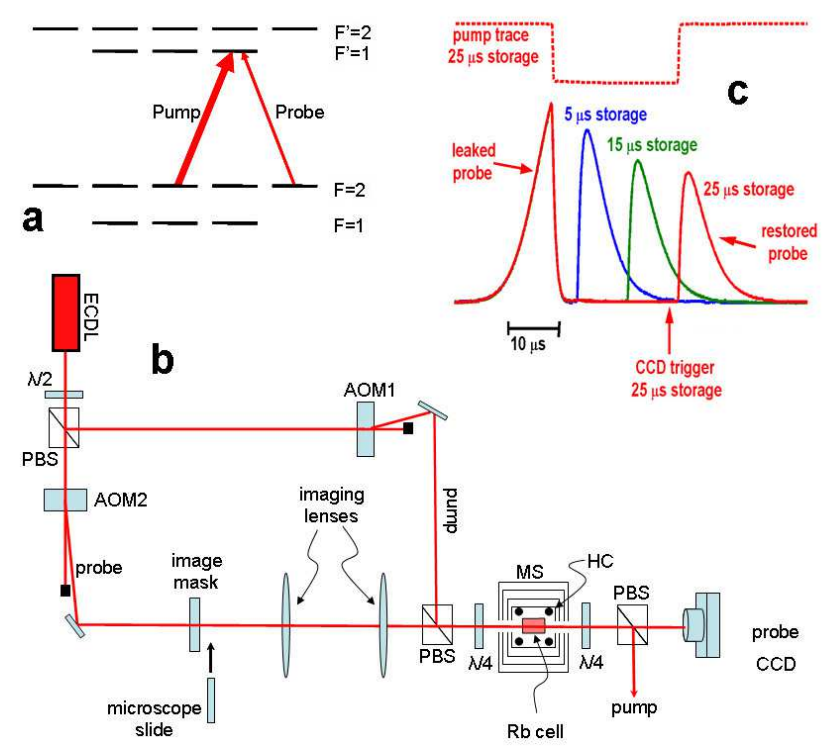

FIG. 1: The experimental setup and sequence. a) The energy level scheme of the ${ }^{87} \mathrm{Rb} \mathrm{D} 1$ transition and the transitions of the pump and the probe. b) The experimental setup: ECDL - external cavity diode laser, $\lambda / 2$ - half wave-plate, PBS polarizing beam splitter, AOM - acousto optic modulator, $\lambda / 4$ - quarter wave-plate, MS - magnetic shield, HC - Helmholtz coils. c) The experimental sequence. The probe is shaped as a temporal Gaussian with $\sigma=5 \mu \mathrm{s}$. After about half of the Gaussian pulse leaves the vapor cell ("leaked probe"), the remaining half is stored in the medium by turning off the pump beam. After a certain storage duration, the pump is turned back on, and the second half of the probe leaves the cell ("restored probe"). The figure shows probe detector traces for storage durations of 5,15 , and $25 \mu$ s and the pump trace for the $25 \mu$ s case.

to diffusion and decreased signal-to-noise ratio due to various decay mechanisms and technical limitations. In order to study quantitatively the limiting factors of the spatial resolution, we stored an image of three resolution lines. Both the lines' thickness and spacing were $340 \mu \mathrm{m}$ at the center of the cell. The left side of Fig. 3 shows the experimental results for storage durations of $2,10,20$, and $30 \mu \mathrm{s}$ (left column) as well as the theoretical prediction (right column). For storage time of $2 \mu \mathrm{s}$, the lines are clearly visible, and, as storage time increases to $30 \mu \mathrm{s}$, the image blurs and its visibility diminishes. The theoretical prediction is based on the assumption that, for a planewave pump, the storage of a weak probe field effectively maps its envelope, $E(\mathbf{r}, 0)$, onto the spatially-dependent slowly-varying ground-state coherence, $\sigma_{b c}(\mathbf{r}, 0)$ [8]. Assuming that the internal state of each individual atom does not change as a result of diffusion, we find that the motion of the atoms during storage can be effectively described by adding a classical diffusion term, $D \nabla^{2} \sigma_{b c}$, to the Bloch equation, where $D$ is the spatial diffusion coef- 


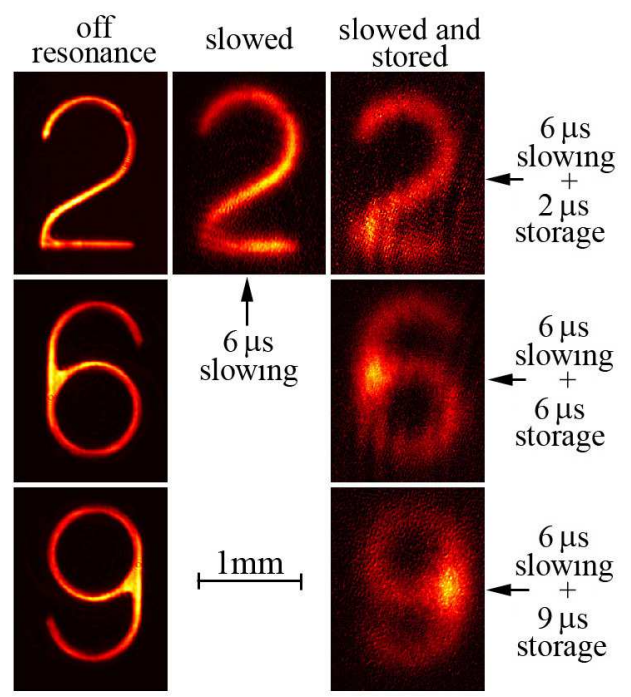

FIG. 2: Images of digits that were slowed and stored for different durations in warm vapor. The left column shows the original images that were to the cell. The middle column shows the image of the digit ' 2 ' that was slowed for about $6 \mu \mathrm{s}$. The right column shows the images after they were stored and retrieved. The images of the digits '2', '6', and '9' were stored for 2,6 , and $9 \mu \mathrm{s}$, respectively. The decay rate in the experiments was about $14000 \mathrm{~s}^{-1}$, and the total intensity in all the experimental data was normalized for the clarity of presentation.

ficient [19]. The ensemble average of the different atoms arriving to the same small macroscopic volume determines the retrieved probe field [20]. Therefore, retrieved fields after storage durations $t$ and $t+\tau$ are related by:

$$
|E(\mathbf{r}, t+\tau)|^{2}=\left|e^{-\frac{\Gamma}{2} \tau} \int d^{3} \tilde{\mathbf{r}}\right| E(\tilde{\mathbf{r}}, t)\left|e^{i \phi(\tilde{\mathbf{r}}, t)} G_{\tau}(\mathbf{r}-\tilde{\mathbf{r}})\right|^{2},
$$

where $G_{\tau}(\mathbf{r})=(2 \pi D \tau)^{-3 / 2} \exp \left[-|\mathbf{r}|^{2} /(4 D \tau)\right]$ is the three-dimensional diffusion propagator, $\Gamma$ is the groundstate decay rate, $E(\tilde{\mathbf{r}}, t)=|E(\tilde{\mathbf{r}}, t)| e^{i \phi(\tilde{\mathbf{r}}, t)}$, and $\phi(\tilde{\mathbf{r}}, t)$ is the phase pattern. Eventually, the initial conditions for the calculation in Fig. 3 are $|E(\tilde{\mathbf{r}}, t=2 \mu s)|$, obtained by taking the square-root of the measured intensity image after storage of $2 \mu \mathrm{s}$. We used $\phi(\tilde{\mathbf{r}}, t)=0$ for the constantphase experiments and an appropriate phase pattern for experiments where the phase of different areas was shifted (see below).

The diffusion of atoms during the storage is evidently the main limit on the storage and retrieval resolution in a medium of warm atoms. A possible method to increase the spatial resolution is based on the fact that the phase pattern of the light field is stored on top of its intensity distribution. By appropriately manipulating the phase of different points in the image, the immunity to diffusion can be improved. In the field of photolithography, a well established technique to reduce the effect of spreading

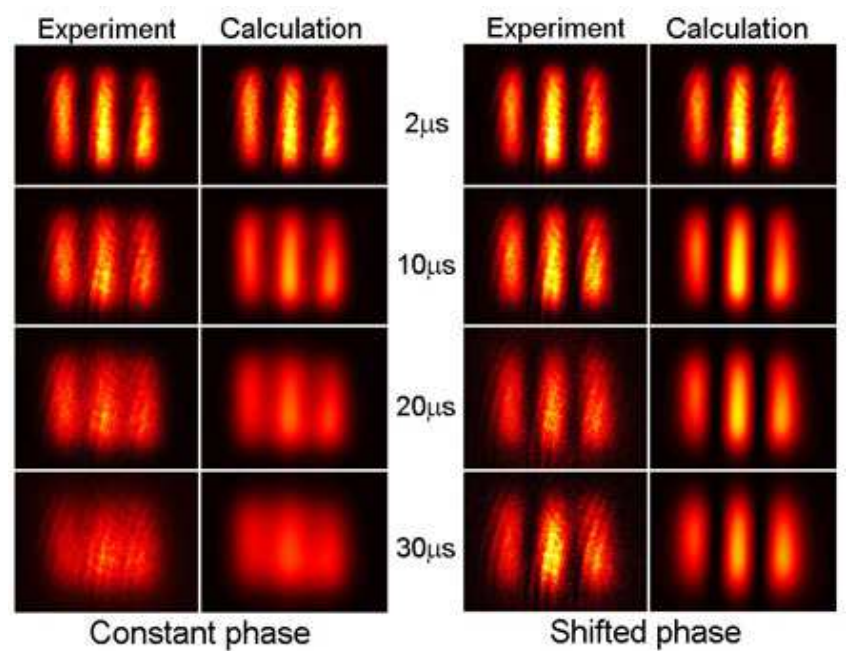

FIG. 3: Storage of an image of three resolution lines for up to $30 \mu \mathrm{s}$. The left hand side shows experimental results (left column) and theoretical prediction (right column), when the image was sent to the cell with a constant phase. A good agreement between the experiment and the calculation was obtained. The right hand side shows the improved resolution achieved when a $\pi$ phase-shift was applied to the two outer lines in the image.

due to diffraction is the so-called phase-shift lithography 12]. Its concept is based on flipping the phases of neighboring features of the image, so that light diffracting to the areas between them will interfere destructively. We implemented an analogous technique in order to mitigate the effect of atomic diffusion. Using microscope slides, we shifted the phase of the two outer lines by $\sim \pi$ with respect to the central line in the probe image. Therefore, the ground-state coherence, created in the atomic medium, had opposite phases in neighboring lines. During storage, atoms of opposite phases diffused to the area between the lines, and the amplitude of the coherence field sum up to zero. Therefore, no probe beam was generated in the forward direction 21], keeping these areas dark in the restored image.

The results of the shifted-phase experiment as well as suitable calculations are depicted in the right side of Fig. 3. The calculation is performed by introducing an appropriate stepwise phase pattern. The dramatic effect of the phase-shift on the visibility of the stored features is pronounced - even after $30 \mu \mathrm{s}$, the lines are well separated, while the respective images in the constant-phase case are completely diffused. These results demonstrate that indeed a complex field is stored in the atomic medium. We have previously demonstrated the topological stability of stored optical vortices to diffusion [18]. The current method is a generalized technique that can be adapted to arbitrary images. Figure 4 shows the measured and calculated visibility of the stored image as a function of 


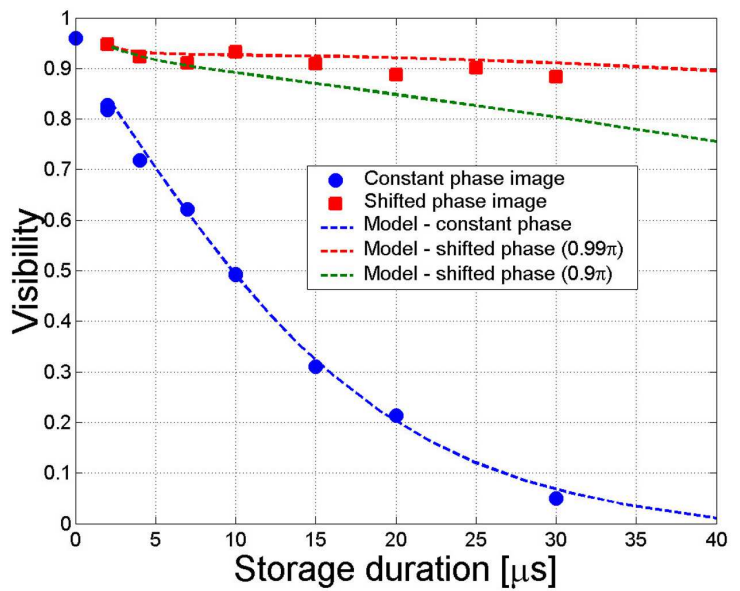

FIG. 4: The visibility of the lines in Fig. 3 as a function of storage duration. The visibility of the constant phase image drops rapidly due to diffusion, while the shifted phase image shows only a small decrease in the visibility. The results are well described by our theoretical model, taking into account inaccuracies in the phase pattern of the phase-shift experiment. An error of $0.01 \pi$ in the phase of the left-most line gives the best fit to the experimental data (red dashed curve). A curve for an error of $0.1 \pi$ is also plotted (green dashed curve) to demonstrate the effect of phase inaccuracies. The visibility presented here is obtained from the two left-most lines, because of a relatively large error in the phase of the right-most line (estimated to be $0.2 \pi$ ).

the storage duration. The rapid decrease of visibility in the constant phase experiment as well as the persistent high visibility in the shifted-phase experiment are well described by our theoretical model.

The phase-shift method can be applied in cases where there is either partial or full a-priori information about the image. In the demonstration above, a prior knowledge regarding the location of the lines in the image allowed us to construct a suitable phase mask. If complete information regarding the image is available, an elaborate phase mask can be designed, even for complicated images, to minimize the effects of diffusion 12. If no apriori information regarding the image is available, other techniques can be used to reduce the effect of diffusion. Increasing the buffer gas pressure is a straight-forward option, limited by the one and two-photon broadening mechanisms. In this case the phase pattern can be used to store information, which might be suitable for certain quantum memory realizations [22, 23]. Another novel method to reduce the effects of diffusion on arbitrary stored images, based on storing the transform plane, was recently investigated theoretically [24], and implemented experimentally [25].

In conclusion, we have demonstrated the ability to store images in warm atomic vapor. The main limitation of the spatial resolution is the diffusion of atoms during the storage. A technique to reduce the effect of diffusion by alternating the phase in neighboring features of the image has been demonstrated by storing $340 \mu \mathrm{m}$ thick lines for as long as $30 \mu \mathrm{s}$, with negligible decrease in their visibility. Our experiment is a trivial case of storing a three-dimensional complex light field, where the shape in the longitudinal (temporal) direction is a simple Gaussian. In principle, it should be possible to store more elaborate temporal shapes, thus storing three dimensional information ("movie").

Helpful discussions with Paz London and Amit Ben-kish are acknowledged. This work was partially supported by the fund for encouragement of research in the Technion.

[1] S. E. Harris, Physics Today 50, 36 (1997).

[2] S. E. Harris and L. V. Hau, Phys. Rev. Lett. 82, 4611 (1999).

[3] M. D. Lukin and A. Imamoğlu, Phys. Rev. Lett. 84, 1419 (2000).

[4] M. D. Eisaman, L. Childress, A. Andre, F. Massou, A. S. Zibrov, and M. D. Lukin, Phys. Rev. Lett. 93, 233602 (2004).

[5] L. M. Duan, M. D. Lukin, J. I. Cirac, and P. Zoller, Nature 414, 413 (2001).

[6] L. V. Hau, S. E. Harris, Z. Dutton, and C. H. Behroozi, Nature 397, 594 (1999).

[7] M. M. Kash, V. A. Sautenkov, A. S. Zibrov, L. Hollberg, G. R. Welch, M. D. Lukin, Y. Rostovtsev, E. S. Fry, and M. O. Scully, Phys. Rev. Lett. 82, 5229 (1999).

[8] M. Fleischhauer and M. D. Lukin, Phys. Rev. Lett. 84, 5094 (2000).

[9] C. Liu, Z. Dutton, C. H. Behroozi, and L. V. Hau, Nature 409, 490 (2001)

[10] D. F. Phillips, A. Fleischhauer, A. Mair, R. L. Walsworth, and M. D. Lukin, Phys. Rev. Lett. 86, 783 (2001).

[11] R. M. Camacho, C. J. Broadbent, I. Ali-Khan, and J. C. Howell, Phys. Rev. Lett. 98, 043902 (2007).

[12] M. D. Levenson, Physics Today 46, 28 (1993).

[13] G. Gilboa, N. Sochen, and Y. Y. Zeevi, IEEE. Trans. Pat. Anal. Machine Intell. 26, 1020 (2004).

[14] A. S. Zibrov, A. B. Matsko, O. Kocharovskaya, Y. V. Rostovtsev, G. R. Welch, and M. O. Scully, Phys. Rev. Lett. 88, 103601 (2002).

[15] O. Firstenberg, M. Shuker, A. Ben-Kish, D. R. Fredkin, N. Davidson, and A. Ron, Phys. Rev. A 76, 013818 (2007).

[16] C. Bolkart, D. Rostohar, and M. Weitz, Phys. Rev. A 71, 043816 (2005).

[17] M. Shuker, O. Firstenberg, R. Pugatch, A. Ben-Kish, A. Ron, and N. Davidson, Phys. Rev. A 76, 023813 (2007).

[18] R. Pugatch, M. Shuker, O. Firstenberg, A. Ron, and N. Davidson, Phys. Rev. Lett. 98, 203601 (2007).

[19] O. Firstenberg, M. Shuker, R. Pugatch, D. R. Fredkin, N. Davidson, and A. Ron, Phys. Rev. A 77, 043830 (2008).

[20] Y. Xiao, I. Novikova, D. F. Phillips, and R. L. Walsworth, Phys. Rev. Lett. 96, 043601 (2006). 
[21] T. Wang, L. Zhao, L. Jiang, and S. F. Yelin, Phys. Rev. A 77, 043815 (2008).

[22] M. Fleischhauer and M. D. Lukin, Phys. Rev. A 65, 022314 (2002).

[23] M. D. Lukin, Rev. of Mod. Phys. 75, 457 (2003).
[24] L. Zhao, T. Wang, Y. Xiao, and S. F. Yelin, Phys. Rev. A 77, 041802 (2008).

[25] P. K. Vudyasetu, R. M. Camacho, and J. C. Howell, Phys. Rev. Lett. 100, 123903 (2008). 